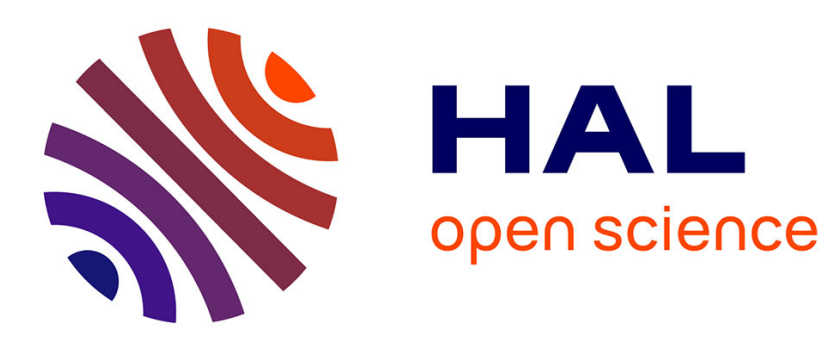

\title{
À propos de l'organisation des pouvoirs dans la région de Potamia aux XIIIe-XVIe siècles \\ Gilles Grivaud
}

\section{To cite this version:}

Gilles Grivaud. À propos de l'organisation des pouvoirs dans la région de Potamia aux XIIIe-XVIe siècles. Cahiers du Centre d'Etudes Chypriotes, 2004, 34, pp.127 - 134. 10.3406/cchyp.2004.1459 . hal-03210953

\section{HAL Id: hal-03210953}

https://hal-normandie-univ.archives-ouvertes.fr/hal-03210953

Submitted on 28 Apr 2021

HAL is a multi-disciplinary open access archive for the deposit and dissemination of scientific research documents, whether they are published or not. The documents may come from teaching and research institutions in France or abroad, or from public or private research centers.
L'archive ouverte pluridisciplinaire HAL, est destinée au dépôt et à la diffusion de documents scientifiques de niveau recherche, publiés ou non, émanant des établissements d'enseignement et de recherche français ou étrangers, des laboratoires publics ou privés. 


\title{
À propos de l'organisation des pouvoirs dans la région de Potamia aux XIIle-XVle siècles Gilles Grivaud
}

\begin{abstract}
Despite the lack of written sources before the Frankish period, we may assert that the Byzantine tradition structures in depth the organization of the Cypriote countryside under the Lusignan as well as during the Venetian domination. This tradition enabled the Lusignan to install a centralized monarchy, the foundation of which was laid on a powerful administrative instrument, the Secrète, whose task was to control the royal domain (the régale ) and the feuds, granted to knights in certain circonstances, such as civil wars and epidemics. The Franks were clustered at Nicosia, what raises the question of the seigniorial domains' organization : in every case, a bailli, appointed by the lord, directly managed the exploitation, with the assistance of Greek or Syrian secretaries who paid the domain's tax to Nicosia. The economical structure thus perfectly reflects the hierarchical organization of the political and administrative powers : it highlights Nicosia as the main outlet for the products of the neighbouring areas which were redistributed and consumed there. Under those circonstances, the fate of the settlements established in the medium Gialias Valley was closely linked to Nicosia, and this for the whole period of Latine domination on the island.
\end{abstract}

\section{Citer ce document / Cite this document :}

Grivaud Gilles. À propos de l'organisation des pouvoirs dans la région de Potamia aux XIIle-XVle siècles. In: Cahiers du Centre d'Etudes Chypriotes. Volume 34, 2004. pp. 127-134;

doi : https://doi.org/10.3406/cchyp.2004.1459

https://www.persee.fr/doc/cchyp_0761-8271_2004_num_34_1_1459

Fichier pdf généré le 19/03/2019 


\title{
À PROPOS DE L'ORGANISATION DES POUVOIRS dans la région de Potamia aux XIIIe-Xvie siècles
}

\author{
Gilles GRIVAUD
}

\begin{abstract}
Despite the lack of written sources before the Frankish period, we may assert that the Byzantine tradition structures in depth the organization of the Cypriote countryside under the Lusignan as well as during the Venetian domination. This tradition enabled the Lusignan to install a centralized monarchy, the foundation of which was laid on a powerful administrative instrument, the Secrète, whose task was to control the royal domain (the régale) and the feuds, granted to knights in certain circonstances, such as civil wars and epidemics. The Franks were clustered at Nicosia, what raises the question of the seigniorial domains' organization: in every case, a bailli, appointed by the lord, directly managed the exploitation, with the assistance of Greek or Syrian secretaries who paid the domain's tax to Nicosia. The economical structure thus perfectly reflects the hierarchical organization of the political and administrative powers: it highlights Nicosia as the main outlet for the products of the neighbouring areas which were redistributed and consumed there. Under those circonstances, the fate of the settlements established in the medium Gialias Valley was closely linked to Nicosia, and this for the whole period of Latine domination on the island.
\end{abstract}

La principale difficulté rencontrée pour apprécier l'histoire de la région de Potamia aux époques franque et vénitienne résulte de situations documentaires contrastées ; au silence complet des rares sources byzantines succèdent quelques mentions éparses sur les villages dans la documentation produite sous les Lusignan. Le $\mathrm{XVI}^{\mathrm{e}}$ siècle bénéficie d'un meilleur éclairage grâce aux archives sauvegardées à Venise et, surtout, grâce au premier recensement ottoman (tahrir), réalisé en 1572, qui offre une image nettement plus concrète de la vie rurale. Cette carence de sources écrites sur la moyenne vallée du Gialias - situation assez fréquente lorsqu'il s'agit de retracer l'histoire des campagnes insulaires ${ }^{1}-n^{\prime}$ interdit pourtant pas de retrouver l'organisation des relations de pouvoir

1. Cf. nos deux études précédentes sur les sources de l'histoire rurale chypriote:

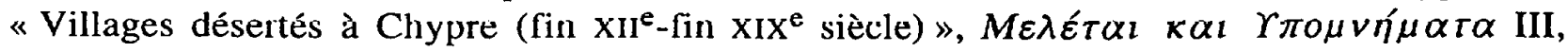
1998, p. 61-132; «Palaichori. A Mountain Village Through the Ages. Historical Review », dans S. Sophocleous (éd.), Palaichoria. Centuries of Heritage, Nicosie, 2002, p. $23-72$. 
dans une région qui ne se distingue pas, par ses structures économiques et sociales, de celles mieux connues par les textes ${ }^{2}$.

Le premier aspect à souligner concerne le poids de l'héritage byzantin dans l'histoire des structures agraires ; certes, les sources byzantines demeurent trop lacunaires pour autoriser une reconstitution de la vie rurale avant le XIII ${ }^{\mathrm{e}}$ siècle ; cependant, l'analyse des textes établis sous le règne des Lusignan ne laisse aucun doute sur la pérennité du régime administratif, social et économique antérieur à 1191. Loin de transformer les institutions byzantines, les Francs les reconduisent et le principe de la consuetudo inspire les relations entre dominants francs et dominés grces, jctant les bases d'un modus vivendi politique et social qui n'est pas bouleversé jusqu'à la conquête ottomane ${ }^{3}$.

L'alliance nouée en 1191 offre un avantage essentiel aux Lusignan puisqu'ils gagnent la collaboration d'une partie des élites locales pour structurer leur État, sur le modèle byzantin ; comme les empereurs de Constantinople, les souverains de Chypre instaurent une monarchie centralisée, qui se réserve plusieurs monopoles économiques, parmi lesquels la frappe de la monnaie, les douanes, la tenue des marchés urbains ${ }^{4}$. C'est encore à Byzance que les Lusignan empruntent le contrôle sur l'exercice de la justice, déléguée à des officiers royaux pour les affaires criminelles, commerciales et toutes celles relevant du droit public ${ }^{5}$. À la différence de la situation observée dans le royaume de Jérusalem ou en Grèce franque, les Lusignan n'ont jamais autorisé des cours de justice seigneuriales et la privatisation du droit qui en découlait. Les structures byzantines ne sont donc pas abrogées : bien au contraire, elles se trouvent combinées, adaptées au nouveau régime politique.

L'héritage byzantin se révèle encore plus prégnant en matière administrative, puisque le corps central de l'administration de la monarchie, la Secrète, conserve la plupart des attributions de l'ancien sekreton tou genikou. Parmi les charges incombant à la Secrète, on note l'établissement d'inventaires cadastraux qui, en français, conservent leur nom grec (prahtico) ; ces inventaires permettent au souverain de connaître l'étendue et les

2. Richard 1947 ; id., "Agricultural Conditions in the Crusader States », dans N. P. Zacour, H. W. Hazard, K. M. Setton (éds), A History of the Crusades V, Madison, 1985, p. 251-294; id., "La seigneurie franque en Syrie et à Chypre : modèle oriental ou modèle occidental ? », dans Actes du $117^{e}$ Congrès National des Sociétés Savantes, Paris, 1993, p. 155-166.

3. Discussion et références dans G. Grivaud, Grecs et Francs dans le royaume de Chypre (1191-1474). Les voies de l'acculturation, mémoire d'habilitation à la direction de recherches, Université Paris I, 2001, troisième partie.

4. P. W. Edbury, The Kingdom of Cyprus and the Crusades, 1191-1374, Cambridge,

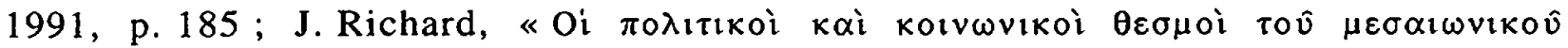
$\beta \alpha \sigma i \lambda \varepsilon i ́ o v »$, dans Papadopoullos (éd.) 1995, p. 350-351; D.M. Metcalf, The White Bezants and Deniers of Cyprus 1192-1285, Nicosie, 1998, p. 3-4; G. Grivaud, "Sur le comerc chypriote de l'époque latine », dans A.A.M. Bryer, G.S. Georghallidès (éds), The Sweet Land of Cyprus, Nicosie, 1993, p. 133-145.

5. P.W. Edbury, op. cit., p. 185 ; J. Richard, op. cit., p. 344-348 et « Tò Sík to tố $\mu \varepsilon \sigma \alpha \imath \omega \nu \imath \kappa o v \hat{~} \beta \alpha \sigma \imath \lambda \varepsilon i ́ o v$ », dans Papadopoullos (éd.) 1995, p. 375-386. 
ressources du domaine royal, la régale, et, surtout, de contrôler les fiefs. La Secrète exerce aussi une compétence fiscale en fixant l'assiette des impôts pesant sur les paysans et les terres de la régale. Par l'étendue de ses qualifications, la Secrète fonctionne comme un organisme centralisant toute la matière féodale; on sait qu'en 1524 ses archives comprenaient 80 volumes. Naturellement, le personnel de la Secrète est en majorité composé de Grecs, les seuls lettrés capables de maîtriser les techniques fiscales byzantines, les mesures, l'arpentage, les usages locaux 6 .

Par scs fonctions, la Secrète tient un rôle fondamental pour asseoir le pouvoir de la monarchie, car celle-ci utilise les registres cadastraux pour accomplir la distribution des fiefs aux chevaliers et les mêmes registres permettent de surveiller les revenus fonciers qui déterminent la nature du service militaire rendu à la couronne. Toute l'armature du système féodal repose donc sur les bureaux de la Secrète et il convient d'insister sur le fait que l'assise territoriale du fief est calquée sur celle léguée par les Byzantins, à travers le chôrion, c'est-à-dire le village considéré comme une unité fiscale cohérente par le fait que son finage est précisément borné et exploité par les paysans du village. En d'autres termes, la seigneurie franque s'est glissée dans le moule byzantin et les Francs ne changent rien à la structure rurale qu'ils trouvent à leur arrivée, conservant toponymie et divisions foncières ${ }^{7}$. Appliquées au cas de Potamia, ces considérations générales expliquent que les villages de la moyenne vallée du Gialias ont conservé leurs noms grecs après 1191 et il est fort probable que leurs finages n'évoluent pas de manière significative de l'époque byzantine à l'époque ottomane.

Cette permanence de la conception fiscale et administrative du chôrion n'implique aucun immobilisme dans le régime des fiefs. Par définition, le fief ne peut être divisé, sous-inféodé, vendu : il reste conçu comme une unité fiscale et militaire inaliénable et ce principe ne sera jamais remis en cause jusqu'en 1570. Par contre, les fiefs peuvent changer de mains ; trois principaux types de domaines sont connus dans le royaume franc : ceux inclus à la régale, ceux attribués par le souverain aux chevaliers à titre de fief, en échange d'un service militaire, enfin ceux accordés aux institutions religieuses ; cette division n'est évidemment pas imperméable car, au fil des guerres civiles, des épidémies et de l'affaiblissement des lignages francs, des fiefs de chevaliers sont réintégrés au domaine royal ${ }^{8}$. De manière logique, pour conserver au fief sa valeur

6. Les deux sources principales pour la connaissance du fonctionnement de la Secrète ont été publiées et étudiées par J. Richard 1983 et G. Grivaud 1992.

7. J. Richard, «La seigneurie franque en Syrie et à Chypre : modèle oriental ou modèle occidental ?", dans Actes du $117^{e}$ Congrès National des Sociétés Savantes, Paris, 1993,

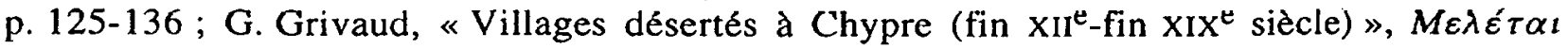

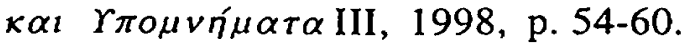

8. Un exemple du contrôle royal sur la circulation des fiefs est particulièrement bien illustré par J. Richard, «Le régime des fiefs à Chypre entre service armé et contribution

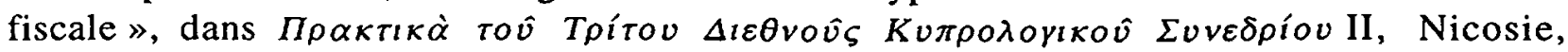
2001 , p. $535-545$. 
militaire et fiscale, la couronne procède à des redistributions de fiefs, notamment lorsqu'elle est confrontée aux crises financières qui se multiplient à partir des années 1360. Parallèlement, afin de limiter la diminution des recettes de son trésor, le roi assigne fréquemment des rentes sur les biens de son domaine.

Ainsi, dans le royaume des Lusignan, les fiefs changent régulièrement de seigneurs, ce dont témoignent les cas qui peuvent être observés dans la moyenne vallée du Gialias : en 1221, Nisou se trouve aux mains de Guillaume Visconte et de sa femme, la vicomtesse de Nephin, et, en 1308, le fief est toujours dans la famille, possession de Raymond Visconte ; plus d'un siècle après, en 1460, le fief, revenu à la régale à une date inconnue, est accordé par Jacques II à l'un de ses partisans, le Sicilien Guillaume Morabito ${ }^{9}$. Le cas de Dali est documenté plus tard, une première fois quand Jacques II l'accorde à Alvise Merie, en 1460, puis quand la veuve du roi, Catherine Cornaro, le concède à son cousin Zorzi Contarini, en 1473, lorsqu'elle l'élève au titre de comte de Jaffa ${ }^{10}$. En revanche, Potamia semble toujours propriété de la régale, au moins en eston assuré par la documentation, de la fin du XIVe siècle au début du XVI ${ }^{\mathrm{e}}{ }^{11}$. Le cas d'Agios Sozomenos est plus complexe, puisque le village est accordé par Jacques II, toujours vers 1460 , en fief libre, à l'un de ses partisans, le Vénéto-Dalmate Nicolò Benedetto ; mais en mars 1468 , le fief est déjà retourné au domaine royal, quand Jacques II assigne à son chancelier, Donato de Aprile, la récolte de blé du village ${ }^{12}$. La longue valse des situations locales démontre que la circulation des fiefs est une réalité permanente des institutions du royaume franc ; elle confirme, surtout, que le souverain est maître de ses fiefs, qu'il distribue et redistribue à son bon vouloir.

On aura relevé que dans la moyenne vallée du Gialias, aucun domaine n'est attribué à un monastère latin ou à l'archevêché de Nicosie, ce qui induit une faible pénétration du rite romain dans la région. Pourtant, une tentative pour fonder une paroisse latine est menée en 1221, à Nisou, sans succès, et cet échec ne constitue pas une surprise puisque seules trois paroisses rurales latines sont dénombrées dans le royaume au $X V^{e}$ siècle ${ }^{13}$. Ce revers illustre la faible implantation de colons francs dans les campagnes et, lorsque les Lusignan veulent stimuler la démographie de leur État, ils

9. Coureas, Schabel (éds) 1997, $\mathrm{n}^{\circ} 42$; Richard 1983, p. 175-176, $\mathrm{n}^{\circ} 148$; R. de Mas-Latrie (éd.), Chroniques d'Amadi et de Strambaldi I, Paris, 1891, p. 285 ; Georges

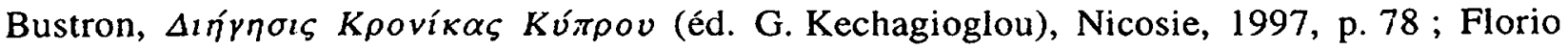
Bustron, p. 394.

10. Florio Bustron, p. 423 ; L. de Mas-Latrie, «Documents nouveaux servant de preuves à l'histoire de l'île de Chypre sous le règne des princes de la maison de Lusignan », Collection des documents inédits: Mélanges historiques IV, 1882, p. 445.

11. L. Machairas, p. 411 et 420 ; Florio Bustron, p. 352 ; L. de Mas-Latrie, Histoire III, p. 525.

12. Florio Bustron, p. 423 ; Richard 1983, n 11.

13. Coureas, Schabel (éds) 1997, $\mathrm{n}^{\circ} 42$; J. Richard, Documents chypriotes des archives du Vatican (XIV et $\mathrm{XV}^{\mathrm{c}}$ siècles), Paris, 1962, p. 73. 
incitent des Francs d'Orient et des chrétiens orientaux à s'installer dans les villes ${ }^{14}$; lorsqu'on rencontre des noms étrangers dans les campagnes, il s'agit presque toujours de Syriens, mais le cas n'est pas avéré dans la zone de Potamia.

L'absence de peuplement franc dans les villages se combine au souci des seigneurs de résider près des centres du pouvoir politique, tous regroupés à Nicosie ${ }^{15}$. En conséquence, on ne trouve que de manière sporadique des bâtiments attestant la présence des maîtres sur leurs domaines, le plus souvent des chapelles privées. Rares demeurent les excmplcs de grands manoirs de campagne, les seuls connus étant ceux des Lusignan, au XIVe siècle : celui d'Aradippou est attesté par les souscriptions de la correspondance d'Hugues IV, ceux de La Cava, près de Nicosie, et de Potamia sont élevés dans le dernier tiers du siècle ${ }^{16}$. Sinon, rien ne laisse supposer que les seigneurs construisent des résidences dignes de leur rang sur leurs domaines, préférant rivaliser en dépenses somptuaires dans leurs palais de la capitale.

La concentration des Francs à Nicosie pose naturellement la question de l'organisation des domaines seigneuriaux. La documentation en dévoile un type particulier, celui groupé autour d'une curia, ensemble de maisons et de bâtiments d'exploitation auquel est attaché un personnel administratif considérable - une dizaine de personnes à Psimolofou en 1318 -, placé sous la direction d'un bailli désigné par le seigneur, auquel une reddition annuelle de comptes est livrée. Un trait remarquable de cette curia tient en l'importance de la réserve seigneuriale qui comprend un septième des terres arables, les autres recettes du domaine provenant de l'élevage, des redevances dues par les paysans, des biens affermés, tels que les moulins et les tavernes-épiceries connues sous le nom de canutes. Enfin, on note le volume important du personnel d'exploitation de la curia, puisqu'à Psimolofou, il rassemble une vingtaine de personnes salariées, parfois pour une durée saisonnière : onze ouvriers agricoles, quatre gardechampêtres, un vignier, un charpentier, un maréchal-ferrant ${ }^{17}$.

Le type de la curia n'est cependant pas universel dans l'île ; il s'avère bien adapté aux villages peuplés disposant d'un terroir assez riche, ce qui est le cas des agglomérations de la moyenne vallée du Gialias. Cependant, un autre modèle d'organisation domaniale existe, connu par un document comptable de 1357, où les trois petits villages de Gauthier de Brienne, dispersés dans l'île, sont administrés par un seul bailli, salarié à haut niveau par son maître. Dans ces villages, les bâtiments d'exploitation sont éparpillés et la main-d'œuvre salariée est remplacée par un groupe d'esclaves. Les recettes des domaines proviennent, comme à Psimolofou, du produit des

14. J. Richard, «Le peuplement latin et syrien en Chypre au XIII siècle», Byzantinische Forschungen 7, 1979, p. 160-162.

15. N. Coureas, G. Grivaud, Chr. Schabel, «The Capital of the Sweet Land of Cyprus: Frankish and Venetian Nicosia ", dans D. Michaelidès (éd.), History of Nicosia, sous presse.

16. L. de Mas-Latrie, Histoire, II, p. 141 ; L. Machairas, p. 411.

17. Richard 1947. 
récoltes, des droits sur les paysans et des biens affermés; compte tenu de l'absence de personnel administratif, une partie des tâches de collecte des redevances est dévolue aux catépans, officiers seigneuriaux le plus souvent recrutés parmi le bas clergé grec ${ }^{18}$.

Dans le cas de la curia de Psimolofou, comme dans celui des villages de Gauthier de Brienne, le seigneur exerce un contrôle direct sur ses domaines par l'intermédiaire d'un bailli, toujours assisté de secrétaires grecs ou syriens. C'est le bailli qui reverse à son maître les produits en nature et les taxes en argent du domaine, qui sont ensuite dirigés vers la résidence du seigneur, c'est-à-dire vers Nicosie, où les surplus sont ensuite écoulés sur les marchés de la capitale ou encore acheminés jusqu'aux ports. À cet égard, il ne semble pas que des marchés seigneuriaux se tiennent à Chypre, laissant les menus échanges nécessaires à l'économie rurale s'accomplir par le biais de l'unique canute villageoise, cette taverne-épicerie propriété du seigneur, généralement affermée à un riche paysan ${ }^{19}$. On constate donc qu'à la hiérarchie des pouvoirs politiques et administratifs répond une structure économique qui consacre Nicosie comme principal centre de consommation et de redistribution des productions agricoles des régions environnantes. Seules Famagouste, Cérines, Limassol et Paphos jouent un rôle équivalent à l'échelon local, mais ces ports ne concurrencent pas Nicosie, où résident le roi et la noblesse.

Dans ces conditions, Nicosie paraît l'unique centre où sont prises les décisions intéressant les destinées des villages de la moyenne vallée du Gialias, à l'époque des Lusignan, et cette situation perdure lorsque Venise s'empare de l'île, en 1474. En effet, cette année-là, soucieux de se concilier la noblesse insulaire, le Sénat vénitien vote la reconduction de tout le corpus de lois coutumières formant la matière féodale ${ }^{20}$. Le changement de statut politique n'altère donc pas les règles de l'organisation féodale, ni le système administratif et les divisions foncières qui la structurent. En revanche, les changements affectent le domaine royal, tombé dans la propriété de la Commune de Venise et administré par le gouvernement colonial. Alors que la monarchie chypriote gérait son patrimoine par un faire-valoir direct, Venise recourt à l'affermage systématique des quelque 300 villages de la reale, l'ancienne régale.

La généralisation de l'affermage a plusieurs conséquences néfastes pour le domaine de la reale. Logiquement, les seules personnes disposant de sommes d'argent importantes appartiennent à la classe des propriétaires fonciers locaux; or ceux-ci s'entendent lors de la mise à l'encan pour maintenir les enchères à bas niveau ; souvent, ils prennent à ferme des villages contigus à leurs domaines personnels, ce qui permet des usurpations de terres ou de main-d'œuvre, évite des investissements dans le bien affermé auquel on préfêre toujours le domaine personnel. Face à ces fraudes, Venise multiplie les enquêtes

18. E. Poncelet, «Comptcs du domaine de Gauthier de Brienne au royaume de Chypre ", Bulletin de la commission royale d'histoire 98, 1934, p. 1-28.

19. Grivaud 1992, p. 587.

20. G. Cozzi, M. Knapton, Storia della Repubblica di Venezia, dalla guerra di Chioggia alla riconquista della Terraferma, Turin, 1986, p. 185. 
fiscales, confiées aux agents de la Secrète, mais le contrôle du domaine public restera un problème récurrent pour le gouvernement colonial ${ }^{21}$.

Venise réagit avec mollesse aux usurpations, car la politique financière de la métropole exige sans arrêt des remises d'argent de la colonie. Dans ces conditions, les fermiers acquittent le plus souvent leurs versements en espèces et non plus en nature, comme il était d'usage jusqu'au début du XVI ${ }^{e}$ siècle. Le besoin d'argent et la dissolution de l'autorité publique sur les terres de la reale ont une conséquence majeure pour l'histoire rurale : les fermiers donnent les terres aux cultures spéculatives, c'est-àdire au coton, au détriment des emblavures, pourtant indispensables à l'équilibre frumentaire de la population insulaire 22 .

Le cas de Potamia illustre assez nettement les contradictions de la politique vénitienne. Intégré au domaine de la reale, le village de Potamia est affermé dès 1476 mais semble de faible rapport puisqu'il est cédé à titre viager, en 1515, à Balian Beltram et à sa femme, pour la somme de 4500 ducats. Il est précisé que la cession n'inclut pas la pleine propriété et que le village retournera à la reale à la mort de Beltram. Pour Venise, il s'agissait d'encaisser une belle somme sans menacer l'intégrité du domaine public puisque Beltram et sa femme n'avaient pas d'enfants et étaient, par ailleurs, âgés respectivement de 55 et 60 ans ${ }^{23}$. Les prévisions des officiers vénitiens se révélèrent exactes, car Beltram et sa femme disparurent peu après et Potamia retourna à la reale, étant ensuite affermé pour une somme annuelle modique, 378 ducats, auxquels s'ajoutaient des rentes en nature, de l'ordre de 400 mozzada de blé et d'orge. Aussi, lorsque Zegno Singlitico propose d'acquérir Potamia pour la somme de 5085 ducats, en 1521, Venise accepte d'emblée l'offre, alors que les caisses de l'État connaissent un sérieux déficit après la deuxième guerre vénéto-ottomane. Fort de sa haute considération dans la hiérarchie nobiliaire, Singlitico obtient la transmission héréditaire du fief, tout en reconnaissant la pleine propriété de la reale. Le statut ambigu de l'opération montre à quel point Venise savait contourner les principes de gestion du domaine public. L'acquisition de Potamia marque pour Zegno Singlitico le début d'une concentration de propriétés foncières, prenant à ferme deux autres prestigieux bailliages des Lusignan, celui d'Aradippou en 1525, pour 10000 ducats, celui de Morfou, en 1528, pour 28500 ducats 24 .

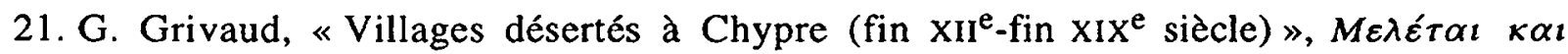

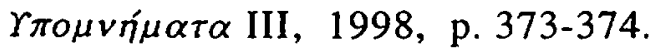

22. Ibid., p. 374-376.

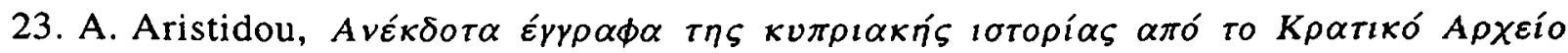

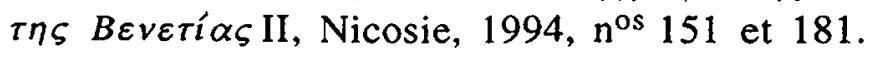

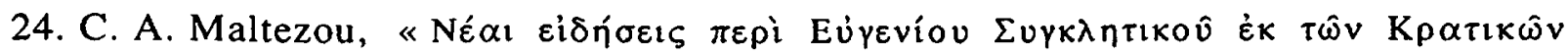

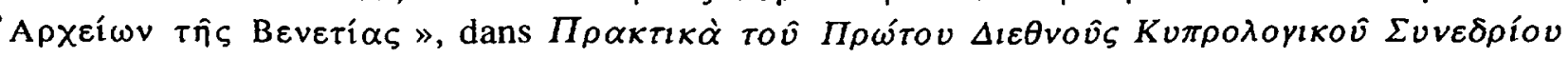
III/A, Nicosie, 1973, p. 233-234 et 238-244 ; B. Arbel, "Greek Magnates in Venetian Cyprus: The Case of the Singlitico Family », Dumbarton Oaks Papers 49, 1995, p. 329 331. 
Le fief de Potamia reste aux mains de la famille Singlitico jusqu'à la conquête ottomane de 1570 et la documentation ne permet guère d'assurer que les Singlitico ont introduit de nouvelles cultures sur leur domaine. Le tahrir de 1572, qui enregistre le montant des taxes par productions agricoles, montre que le finage de Potamia est principalement consacré à l'arboriculture (44\%), la culture du coton et celle des céréales fournissant chacune $24 \%$ des taxes 25 . Si le coton visait la satisfaction des marchés vénitiens, l'arboriculture était sans doute destinée à approvisionner les marchés de Nicosie et il est fort probable que cette orientation avait été dessinée par les Lusignan, si on se réfère à la description du manoir de Potamia consignée par Luchino del Campo, en $1412^{26}$. À nouveau, l'attraction économique de Nicosie semble déterminante dans les choix accomplis par les seigneurs du village. Ainsi, les centres de pouvoir à Potamia, pour toute la période franque et vénitienne, sont regroupés à Nicosie, ce qui s'explique, en partie, par la proximité de la capitale mais aussi parce que tous les rouages des administrations publiques et privées y sont concentrés.

GRHIS, Université de Rouen

\section{BIBLIOGRAPHIE}

Coureas (N.), Schabel (C.), éds, 1997, The Cartulary of the Cathedral of the Holy Wisdom of Nicosia, Nicosie.

Florio Bustron, Chronique de l'île de Chypre (éd. R. de Mas-Latrie), Paris, 1886.

Grivaud (G.), 1992, «Ordine della Secreta di Cipro. Florio Bustron et les institutions franco-byzantines afférentes au régime agraire de Chypre à l'époque

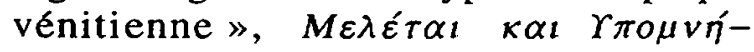
$\mu \alpha \tau \alpha$ II, p. 531-592.

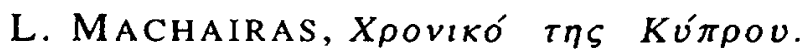

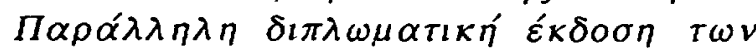
$\chi \varepsilon \iota \rho \circ \gamma \rho \alpha \dot{\phi} \omega v$, M. Pieris, A. NikolaouKonnari (éds.), Nicosie, 2003.
MAS-LATRIE (L. de), 1852-1861, Histoire de l'île de Chypre sous le règne des princes de la maison de Lusignan, I-III, Paris.

PAPAdOPOUllos (Th.), éd., 1995, 'Iotopía tĥs Kútoov IV, Nicosie.

R ICHARD (J.), 1947, «Le casal de Psimolofo et la vie rurale en Chypre au $\mathrm{XIV}^{\mathrm{e}}$ siècle", Mélanges d'Histoire et d'Archéologie de l'École française de Rome 59, p. 121-153.

RICHARD (J.), 1983, Le Livre des Remembrances de la Secrète du royaume de Chypre (1468-1469), Nicosie.

25. H. Inalc1k, "Ottoman Policy and Administration in Cyprus after the Conquest »,

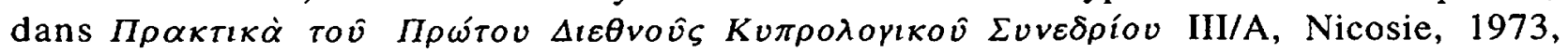
p. $134-135$.

26. G. Grivaud, Excerpta Cypria Nova. Voyageurs occidentaux à Chypre au $\mathrm{Xv}^{\mathrm{e}}$ siècle, Nicosie, 1990 , p. 42 et 48. 